\title{
Assessment of uncertainties in projecting future changes to extreme storm surge height depending on future SST and greenhouse gas concentration scenarios
}

\author{
Jung-A Yang ${ }^{1,4}$ D $\cdot$ Sooyoul $\mathrm{Kim}^{2} \cdot$ Sangyoung Son $^{3} \cdot$ Nobuhito Mori $^{4} \cdot$ Hajime Mase $^{4}$
}

Received: 3 November 2019 / Accepted: 25 June 2020/Published online: 10 July 2020

(C) The Author(s) 2020, corrected publication 2020

\begin{abstract}
We assess uncertainties in projecting future changes in extreme storm surge height (SSH) based on typhoon data extracted from ensemble experiment results with four sea surface temperature (SST) conditions and three global warming scenarios using a single atmospheric global climate model. In particular, this study focus on typhoons passing around the Korean Peninsula (KP) defined as the region of 32 to $40^{\circ} \mathrm{N}$ and 122 to $132^{\circ}$ E. It is predicted the number of the typhoons affecting the KP will decrease by $4 \sim 73 \%$ while their strength will increase by $0.8 \sim 1.4 \%$ under the given future conditions. The locations of genesis and lysis of the typhoons are expected to be shifted towards the northwest and northeast for all ensemble experiment conditions, respectively. However, the extent of their change varies depending on the future SST and global warming conditions. Storm surge simulations were carried out by using predicted typhoon data as an external force. It is found that future SST patterns and climate warming scenarios affect future typhoon characteristics, which influences values of extreme SSH and locations of the vulnerable area to storm surge under the future climate conditions. In particular, the values of extreme SSH and the locations of the vulnerable area to storm surge appear to be strongly influenced by both pathway and frequency of intense typhoons.
\end{abstract}

Keywords Extreme storm surge height $\cdot$ Climate change $\cdot$ Ensemble experiment $\cdot$ Sea surface temperature - Greenhouse gas concentration scenario

Electronic supplementary material The online version of this article (https://doi.org/10.1007/s10584-02002782-7) contains supplementary material, which is available to authorized users.

Jung-A Yang

yangja.1985@gmail.com

Nobuhito Mori

https://orcid.org/0000-0001-9082-3235

Extended author information available on the last page of the article 


\section{Introduction}

Tropical cyclone (TC) projections are summarized in the IPCC (2019) as follows: (1) the proportion of TCs that reach category 4-5 levels will increase (medium confidence); (2) global TC frequency will change, although most modeling studies project some decrease in global TC frequency (low agreement, medium evidence); and (3) sea level rise will lead to higher storm surge levels for the TCs, assuming all other factors are unchanged (very high confidence). The starting point of the manuscript is based on item 3 of the SROCC classification above. The changes in TC frequency depend on climatological environment (e.g., wind shear or humidity) and other factors in the low latitude and the changes in TC intensity depend on increasing SST and atmospheric stability and other factors in the low to middle latitude. The uncertainty of these projections is still large and diverged but it is important to project extreme storm surge levels based on current knowledge of projections for adaptation. Storm surge is an abnormal sea level rise due to low pressure and strong winds in tropical cyclones (including typhoons) or extratropical cyclones. The occurrence of storm surge is not only depending on TC intensity but is also depending on TC angle to bay and translation speed. For grounded policy-making of coastal zone protection against the storm surge in the future climate condition, it is necessary to identify storm surge-prone areas on national or regional scales in the future climate (Troccoli et al. 2012; Lapidez et al. 2015; Vousdoukas et al. 2016).

The Korean Peninsula (KP) which is located in East Asia is no exception. The extreme SSH is one of the important parameters for designing the crest height of coastal structures in the Korean Peninsula (Ministry of Oceans and Fisheries 2017). To mitigate the storm surge risk, many studies have projected future potential extreme SSH on the KP coast. However, their interests focused on one or a few specific points in the KP (e.g., Hur et al. 2006; Yang and Kim 2014). There are only few studies on the extreme SSHs for regional scales (e.g., Kim et al. (2014)). It is essential to estimate future storm surge risks and its uncertainties for safely managing coastal zone and efficiently designing coastal structures (e.g., Park et al. 2011; Ceres et al. 2017; Lin et al. 2019). Therefore, the present study aims to assess the uncertainties in projecting the future change of potential extreme SSHs along the KP using the typhoon information which was extracted from ensemble experiment results of a single atmospheric general circulation model with a resolution of $60 \mathrm{~km}$ established by the Japanese Meteorological Research Institute (denoted as MRI-AGCM3.2H; Mizuta et al. 2012).

When projecting future changes of TC by using the atmospheric general circulation model (hereafter, AGCM), there are several uncertainties due to model resolution, model physics parameterization, given sea surface temperature (SST) under future climate condition, and global warming scenarios (e.g., Bengtsson et al. 2007; Stowasser et al. 2007; Walsh et al. 2010;). The range of uncertainties can be accordingly different for each source and need to clarify. Regarding uncertainty caused by AGCM resolution and physical parameterization of MRI-AGCM3.2, some recommendations have been proposed through the analysis of model accuracy in global and ocean basin scales. Firstly, for model resolution, Murakami and Sugi (2010) investigated its effects on the projection of TC features by comparing results from four different mesh sizes-180, 120, 60, and $20 \mathrm{~km}$. Each resolution was employed for both present-day (1979-2003) and global warming (2075-2099) projections. Each projection was prescribed either by observed SST and or by predicted future SST based on the ensemble mean of 18 models in the Intergovernmental Panel for Climate Change Fourth Assessment Report under A1B Scenarios. The model accuracy for mesh sizes was verified based on the presentday projection. Relatively finer resolutions of $60 \mathrm{~km}$ and $20 \mathrm{~km}$ presented a significant 
increase in the frequency of future intense TCs, whereas coarser resolutions larger than $60 \mathrm{~km}$ showed less changes. The highest resolution of $20 \mathrm{~km}$ produced the best outputs in accuracy as to TC properties such as the intensity and the genesis number. These results also indicated that the $60-\mathrm{km}$ mesh is regarded as the critical resolution when assessing the future change of the TC. Another source of uncertainty, the model physical parameterization was examined by Kitoh and Endo (2016) who found the cumulus convection scheme among physical processes is a key factor causing the uncertainty, as the TC involves convective systems. Mizuta et al. (2012) conducted single-model multi-physics ensemble simulations using MRI-AGCM with three cumulus convection schemes: Yoshimura scheme (YS), Arakawa-Schubert scheme, and Kain-Fritsch scheme. They concluded that the YS scheme produces tropical precipitation more realistically than the Arakawa-Schubert scheme in MRI-AGCM.

Few studies, on the other hand, have assessed the uncertainties in projecting future changes in TC resulting from future SST conditions and global warming scenarios. It is reported that the different global warming scenarios result in quite dissimilar characteristics of future climate change patterns, and the different SST condition also gives rise to diverse climate impacts in the global and ocean basin scales (Murakami et al. 2012a; Mizuta et al. 2014; Kitoh and Endo 2016). The uncertainties stemming from physics and numerical modeling configuration can be reduced by improving the accuracy of AGCMs, while those from the global warming scenario and future SST condition cannot be reduced. Besides the uncertainties from physical and numerical parameters of AGCMs, the effects of the global warming scenario and spatial pattern of future SST on the projection of future TCs are also quite important for climate change impact assessment. Moreover, the uncertainty in projecting future TC properties varies according to scale and location of the region of interest (Murakami et al. 2012a, 2012b). In order to evaluate the future storm surge risk at the national scale, it is necessary to evaluate typhoon characteristics for a specific country prior to conducting storm surge simulation using them.

The present study assesses the uncertainties in projecting future SSH using the typhoon data which is extracted from ensemble experiment results of MRI-AGCM3.2H with three global warming scenarios and four future SST conditions. First, the reproducibility of typhoon data is evaluated by making a comparison between modeled typhoon data and observed one, and then, their future change is discussed. Second, storm surge simulation using the typhoon data as the external forcing is conducted to project potential extreme SSH around the KP. Finally, uncertainties in projecting the potential extreme SSHs depending on future SST conditions and global warming scenarios are estimated. This paper is divided into three sections. A brief introduction of ensemble experiment conditions, the typhoon data, and storm surge simulation setup are given in Section 2. Section 3 discusses the typhoon characteristics passing around the KP for present and future climate conditions and shows future extreme SSHs around the KP coast. The last section contains the conclusion with a summary of this study as well as future research plans.

\section{Methodology}

\subsection{Ensemble experiments and future climate conditions}

A series of ensemble experiments using MRI-AGCM3.2H, which is one of the models in CMIP5, were conducted by different research groups (refer to Kitoh et al. 2016). To evaluate 
the effects of the global warming scenario which is represented by representative concentration pathways (RCP; van Vuuren et al. 2011) and future SST change patterns on the projection of future SSHs, the present study used the output of seven ensemble experiments with the YS scheme (Table 1). Each ensemble experiment used in this study was abbreviated for convenience (Table 1): HPA and HFA denote experiments under present and future climate conditions, respectively. The sequence of letters in the abbreviations following HFA indicate the conditions of the global warming scenario and the spatial pattern of SST. A series of climate experiment was conducted for the present climate condition, and RCP2.6, RCP4.5 and RCP8.5 in the future climate conditions. As AGCM needs to use SST as forcing, four different SSTs (denotes SST cluster 0 to 4 ) were given to future climate conditions, respectively. The four SSTs are consist of cluster analysis for 28 models in CMIP5 (see Fig.2 of Mizuta et al. (2014) in detail).

SST cluster 0 is a mean of the CMIP5 model and the remaining three SST patterns have the following meaning. The tropical SST changes patterns were estimated differently for each model in CMIP5 (Christensen et al. 2013). This might affect the tropical circulation changes which result in the changes in TC genesis. Mizuta et al. (2014) aimed to examine the difference of the tropical SST change pattern among several models in CMIP5. By conducting the cluster analysis defined in Murakami et al. (2012a) and Endo et al. (2012), they classified the future change patterns of annual-mean tropical SST projected by the models. The cluster analysis was carried out on both historical and RCP8.5 scenario experiments. The SSH change patterns were categorized into three types as cluster 1, cluster 2 , cluster 3 by averaging each cluster after normalized by the SST change of the annual tropical mean $\left(30^{\circ} \mathrm{S}-30^{\circ} \mathrm{N}\right)$. Each cluster was comprised of the SST changes of 8,14 , and 6 models, respectively. It was found that three types of the clustered SST change patterns are a close parallel to SST for tropical and global means (refer to Fig.2 of Mizuta et al. (2014)). It is expected that the annual-mean SST will increase tropically and almost globally showing the zonal gradient of the change. However, cluster 1 shows that warming of annual-mean SST in the Northern Hemisphere is larger than that of the Southern Hemisphere. In particular, the greatest warming takes place in the northern North Pacific. This spatial distribution is more prominent in cluster 2. In contrast, there is substantial warming in the western North Pacific. The clustered SST patterns for other RCP experiments were made by applying the results of the RCP8.5 experiments. Correlation coefficients of the SST patterns among the different experiments are higher than 0.91 except for those between RCP2.6 and RCP8.5 experiments (0.76).

Table 1 Conditions of ensemble experiments using a single atmospheric general circulation model with resolution of $60 \mathrm{~km}$ established by the Japanese Meteorological Research Institute, denoted as MRI-AGCM3.2H

\begin{tabular}{|c|c|c|c|c|}
\hline Climate condition & Calculation period & SST pattern & Greenhouse gas emission scenario & Abbreviation \\
\hline Present & 1984-2003 & HadISST1.1 & Present & HPA \\
\hline \multirow[t]{6}{*}{ Future } & 2080-2099 & CMIP5 mean & RCP 2.6 & HFA-c0-rcp26 \\
\hline & & CMIP5 mean & RCP 4.5 & HFA-c0-rcp45 \\
\hline & & CMIP5 mean & RCP 8.5 & HFA-c0-rcp 85 \\
\hline & & CMIP5 cluster 1 & RCP 8.5 & HFA-c1-rcp 85 \\
\hline & & CMIP5 cluster 2 & RCP 8.5 & HFA-c2-rcp85 \\
\hline & & CMIP5 cluster 3 & RCP 8.5 & HFA-c3-rcp85 \\
\hline
\end{tabular}




\subsection{Typhoon data}

Typhoon data, which were extracted from the results of each ensemble experiment by applying the TC detection technique (Murakami et al. 2012a), was employed to project the future SSHs around the KP. This study focused on typhoons passing around the KP which is defined as the region of 122 to $132^{\circ} \mathrm{E}$ and 32 to $40^{\circ} \mathrm{N}$. These typhoon data include information such as typhoon occurrence time, location, central pressure, and wind speed. In storm surge simulation, the typhoon occurrence time, the location, and the central pressure were used to produce atmospheric fields of pressure and wind for each typhoon event.

\subsection{Storm surge modeling}

A series of storm surge simulations was performed using the coupled surge, wave, and tide model (SuWAT; Kim et al. 2008). A couple of nested domains can simultaneously run in the SuWAT model using the message passing interface. The SuWAT consists of three modules of tide-surge, wave, and typhoon that are integrated into each domain. In the present study, the tide-surge and typhoon modules were used. The tide-surge module is based on the depthintegrated non-linear long-wave equations discretized in space on the staggered Arakawa $\mathrm{C}$ grid and in time using the leap-flog method. Tide and flooding were not considered. Parametric typhoon models are internally integrated into SuWAT as the typhoon module predicts the atmospheric fields of pressure (sea level pressure) and wind (U10) as a meteorological boundary condition to force surges. Spin-up periods of $48 \mathrm{~h}$ are included prior to each simulation for the stability of simulations.

Of parametric wind and pressure models embedded in SuWAT, the Fujita (1952) and Fujii and Mitsuta (1986) models were adopted to estimate the pressure and wind, respectively. The parametric wind and pressure model requires information on the typhoon characteristic such as the typhoon location (longitude and latitude), the central atmospheric pressure, the radius to the maximum wind speed from the typhoon center $\left(R_{\max }\right)$, and the moving speed to generate the pressure and wind field. However, the used typhoon data does not provide the maximum wind radius $\left(R_{\max }\right)$ among the typhoon characteristics, so that an empirical formula by Yasuda et al. (2010) was used to estimate it. They proposed the formula which statistically estimates the typhoon radius with the central atmospheric pressure based on the estimated typhoon radius data from synoptic weather charts and the observed central pressure data from best track data from 1951 to 2000 by JMA.

The calculation domains were downscaled in three steps as shown in Fig. 1. The outermost domain (D1) covers the area from 117 to $132^{\circ} \mathrm{E}$ and 20 to $42^{\circ} \mathrm{N}$. The innermost domain (D4) is the area 127 to $130^{\circ} \mathrm{E}$ and 33.5 to $35.75^{\circ} \mathrm{N}$ with a 30 -s spatial resolution (approximately $760 \mathrm{~m}$ ). Here, this domain is defined as the southeastern region of the KP. More detailed information on each calculation domain is given in Supplementary Table 1 . The bathymetry data of the General Bathymetric Chart of the Oceans with 1-min spatial resolution was employed to the outermost domain. For the rest of the domains, the bathymetric dataset with a 30-s spatial resolution (Seo 2008) was used. The time step for computation was $300 \mathrm{~s}$.

Yang et al. (2017) verified the SuWAT model performance as hindcasting six typhooninduced SSHs $1.0 \mathrm{~m}$ higher around the southeastern coast of the KP. In Yang et al., by comparing the simulated maximum SSHs with the observed ones available from tidal stations, it was found that the SSHs tend to be overestimated under the following conditions: (1) the tidal station is located to the right side of the typhoon, and (2) the tidal station is close to the 


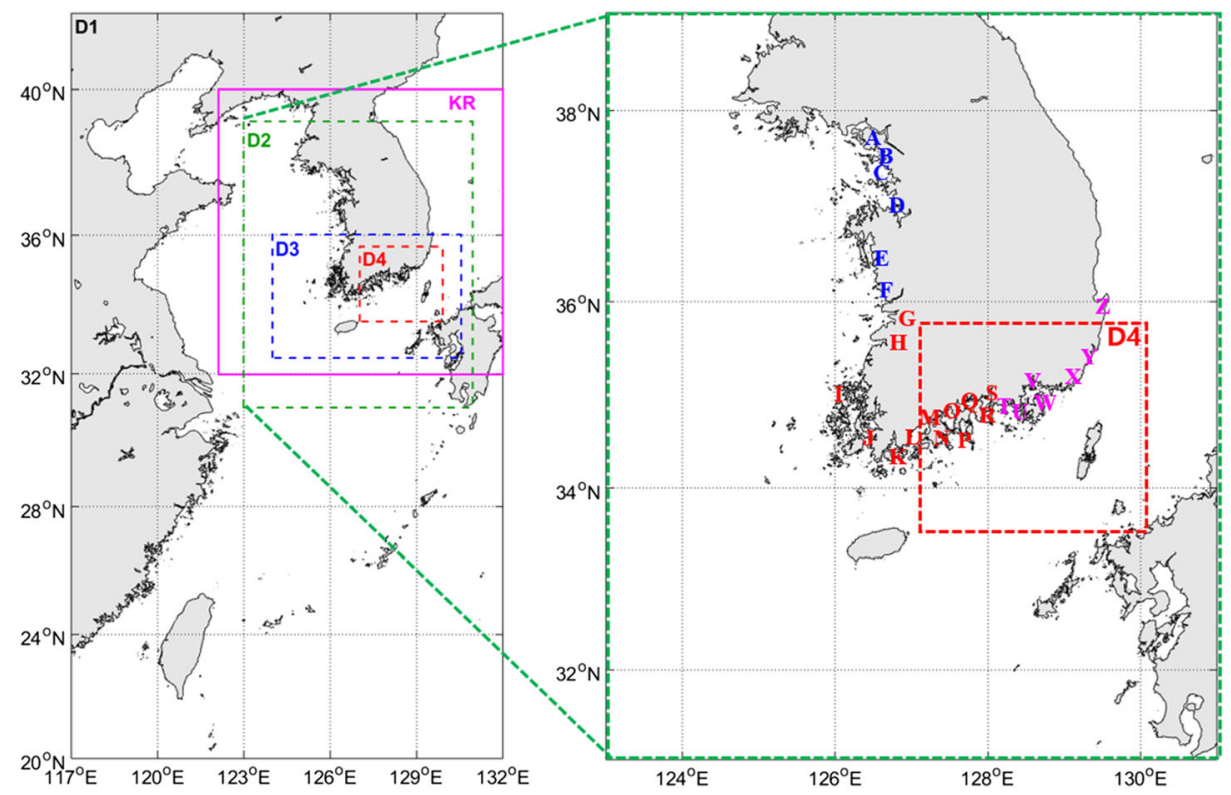

Fig. 1 Computation four domains of storm surge simulation. The notation of "\#” in "D\#” and "KR" in the left panel mean the domain number and a definition region for typhoons passing in the vicinity of the Korean Peninsula, respectively. The letters A through $\mathrm{Z}$ in the right panel represent the name of coastal cities in the KP and their colors of blue, yellow, and purple mean specifically the cities on the west coast, on the south coast, and southeastern coast of the KP: (A) Ganghwa, (B) Incheon, (C) Ansan, (D) Asan Bay, (E) Boryeong, (F) Seocheon, (G) Gunsan, (H) Buan, (I) Sinan, (J) Haenam, (K) Wando, (L) Jangheung, (M) Boseong, (N) Goheung, (O) Suncheon, (P) Yeosu, (Q) Gwangyang, (R) Namhae, (S) Sacheon, (T) Goseong, (U) Tongyeong, (V) Changwon, (W) Geoje, (X) Busan, (Y) Ulsan, (Z) Pohang

typhoon. This tendency is more pronounced at the tidal station with a complex coastline. On the other hand, the tidal station is located sufficiently far from the center; the SSHs have a tendency to be underestimated irrespective of the relative position between them. For solving the model-induced bias, they proposed the model bias correction method considering a geographic feature, especially coastline complexity. It was validated that the bias correction method can improve model accuracy by $23 \%$. However, the present study does not apply the model bias correction method proposed by Yang et al. (2017), because this study considers future changes in extreme SSHs. The future changes were estimated using the difference between future projection and historical simulation. We assumed that model biases existing in both the historical simulation and future projection are time invariant (e.g., Buser et al. 2009; Pickering et al. 2017). So, the model biases are canceled out when calculating the future change to the extreme SSHs.

\section{Results and discussion}

\subsection{Changes in typhoon characteristics}

As mentioned in Section 1, simulated typhoons by the AGCMs have bias for intensity and frequency due to the limited of numerical modeling. Therefore, bias correction for simulated 
typhoons is important prior to conducting their impact assessments. In this section, typhoon properties such as occurrence frequency, track, and intensity were verified by comparing them with observed typhoon dataset (International Best Track Archive for Climate Stewardship, IBTrACS; Knapp et al. 2010)under the present climate condition. In addition, future changes in the typhoon characteristics were also discussed by comparing the results under present and future climate conditions. As stated above, we focused on typhoons passing around the KP which is defined as the region of 122 to $132^{\circ} \mathrm{E}$ and 32 to $40^{\circ} \mathrm{N}$ (refer to the solid area with purple color in Fig. 1).

\subsubsection{Typhoon occurrence frequency}

First, characteristics of the typhoon occurrence frequency were examined. The total occurrence frequency (annual average occurrence frequency) of the typhoons affecting the KP in the present climate condition was found to be 74 (3.7 per year) and 26 (1.3 per year) for IBTrACS and HPA, respectively. It was found that the present climate experiment using MRIAGCM3.2H underestimates both the total occurrence frequency and the annual average occurrence frequency of the typhoons (Table 2 and Supplementary Fig. 1). It should be noted that the TC detection technique applied in the present study was set to match up the total TC genesis frequency with observed ones in global and ocean basin scales. Although the total TC genesis frequency of HPA using MRI-AGCM3.2H on the global scale and the Western North Pacific basin scale are largely similar to those of IBTrACS (Murakami et al. 2012a), the total TC occurrence frequency on the national scale, especially in the KP, exhibits a discrepancy. It could result from some of the simulated typhoons that readily disappear before approaching the KP. These results imply that verification of typhoon properties should be preceded when utilizing the typhoon data from climate experiments for future disaster assessments on a national scale. This TC occurrence frequency bias should be considered significant for projecting the typhoon frequency under the future climate condition. The future change in the typhoon frequency for each ensemble experiment is estimated by subtracting the typhoon frequency under the present climate condition from that under the future climate condition. It was assumed that the frequency bias would be offset in calculating future changes of the typhoon frequency.

The total typhoon occurrence frequency in each climate experiment under the future climate condition was 25, 20, 14, 7, 16, and 16, in HFA-c0-rcp26, HFA-c0-rcp45, HFA-c0-rcp85,

Table 2 Characteristic values of typhoon properties of observation (IBTrACS) and simulations using MRIAGCM3.2H

\begin{tabular}{|c|c|c|c|c|c|}
\hline \multirow{2}{*}{$\begin{array}{l}\text { Climate } \\
\text { condition }\end{array}$} & \multirow{2}{*}{$\begin{array}{l}\text { Abbreviation for } \\
\text { experiments }\end{array}$} & \multicolumn{2}{|c|}{ TC occurrence } & \multicolumn{2}{|c|}{ Minimum central pressure (intensity) } \\
\hline & & Frequency & $\begin{array}{l}\text { Its future decreasing } \\
\text { rate }(\%)\end{array}$ & $\begin{array}{l}\text { Mean values } \\
(\mathrm{hPa})\end{array}$ & $\begin{array}{l}\text { Its future decreasing } \\
\text { rate }(\%)\end{array}$ \\
\hline \multirow[t]{2}{*}{ Present } & Observation & 74 & - & 981.6 & - \\
\hline & HPA & 26 & - & 981.6 & - \\
\hline \multirow[t]{6}{*}{ Future } & HFA-c0-rcp26 & 25 & 4 & 974.0 & 0.8 \\
\hline & HFA-c0-rcp45 & 20 & 23 & 970.7 & 1.1 \\
\hline & HFA-c0-rcp85 & 14 & 46 & 968.6 & 1.3 \\
\hline & HFA-c1-rcp85 & 7 & 73 & 968.1 & 1.4 \\
\hline & HFA-c2-rcp85 & 16 & 38 & 969.1 & 1.3 \\
\hline & HFA-c3-rcp85 & 16 & 38 & 969.1 & 1.3 \\
\hline
\end{tabular}


HFA-c1-rcp85, HFA-c2-rcp85, and HFA-c3-rcp85, respectively. The total typhoon occurrence frequency under the future climate conditions tends to decrease as greenhouse gas concentrations increase. While there was no big difference in the typhoon occurrence frequency according to the SST pattern, only the HFA-c1-rcp85 showed a noticeable lower occurrence frequency.

The future change rate of the typhoon occurrence frequency $\left(N_{\text {future change }}, \%\right)$ was calculated as

$$
N_{\text {future change }}=\frac{N_{\text {future }}-N_{\text {present }}}{N^{\text {present }}} \times 100
$$

where $N_{\text {present }}$ and $N_{\text {future }}$ are the occurrence frequency of the typhoons in present and future climate conditions, respectively. It was expected that the typhoon frequency affecting the KP was significantly reduced in the range of 4 to $73 \%$ depending on the future climate condition. Looking at this range in detail by future climate conditions, the reduction rates are in the ranges of 4 to $46 \%$ for the global warming scenarios and 38 to $73 \%$ for the SST condition, respectively. As global warming intensity increased with RCP2.0, RCP4.5, and RCP8.5, the future changes in typhoon frequency decreased to 4\%, 23\%, and 46\%. Murakami et al. (2012a) projected future changes in TC frequency in the Western North Pacific region under the A1B emission scenario using MRI-AGCM with spatial resolutions of $20 \mathrm{~km}, 60 \mathrm{~km}$, and $120 \mathrm{~km}$, respectively. A1B has a global warming scale similar to that of RCP 6.0 (van Vuuren and Carter 2013). They reported that the TC frequency would decrease by 35 to $38 \%$. The typhoon frequency in the KP region appears to decrease in proportion to the global warming intensity. On the other hand, the future change of the typhoon frequency according to SST conditions has a large deviation. The c0, c2, and c3 of the SST conditions show 46\%, 38\%, and 38\% reduction, respectively, whereas $\mathrm{c} 1$ alone indicates a big difference of $73 \%$. But, given SST conditions we used are representative values of some of AOGCM projection, it is difficult to directly analyze the relationship between SST and the number of typhoons affecting the KP. In addition, a general relationship between SST and TC tracks is currently unknown (e.g., Ren et al. 2014). Further studies should be required to investigate this.

\subsubsection{Typhoon track}

Next, the characteristic of typhoon tracks was assessed. Figure 2 shows tracks, genesis, and lysis locations of typhoons under present and future climate conditions. Mean genesis locations of the typhoons (longitude, latitude) for IBTrACS and HPA are $\left(138.16^{\circ}, 15.66^{\circ}\right)$ and $\left(136.37^{\circ}, 16.64^{\circ}\right)$, respectively. The differences between those ( $\Delta$ longitude, $\Delta$ latitude) are calculated to be $\left(1.79^{\circ},-0.98^{\circ}\right)$, where positive values denote relative east and north for longitude and latitude, respectively. Meanwhile, mean lysis locations of the typhoons for IBTrACS and HPA are found to be $\left(144.49^{\circ}, 42.40^{\circ}\right)$ and $\left(138.10^{\circ}, 40.20^{\circ}\right)$, respectively, with a difference of $\left(6.39^{\circ}, 2.20^{\circ}\right)$ in between them. The bias of the lysis locations between observation and simulation was larger than the one in the genesis locations. These errors are thought to be originating from the limitations of the model. Since MRI-AGCM3.2H has a limitation to reproduce typhoons above $40^{\circ}$ north latitude, a relatively large error in the lysis location can be introduced. However, as shown in Fig. 2 a and e, typhoon tracks based on HPA was qualitatively in good agreement with observations: for example, the typhoons were generated in the area of 10 to $20^{\circ} \mathrm{N}$ and 120 to $160^{\circ} \mathrm{E}$, and then they headed for northwest towards Taiwan. 
Thereafter, they changed their trajectory near Taiwan to the northeast and approached the southwest coast of the KP.

Figure $2 \mathrm{~b}, \mathrm{c}, \mathrm{d}$, and h show comparisons of the typhoon tracks obtained from the ensemble experiments using the different spatial patterns of SST under the same global scenario, RCP8.5. In all experiments, the genesis and lysis locations of typhoon were expected to move to the northwest under the future climate condition while the paths were altered depending on the spatial pattern of SST during each typhoon period. In HFA-c3-rcp85, the typhoons changed their tracks to the northeast in the vicinity of Taiwan, just like the present climate experiment, and then, some of them turned north again in the Korea/Tsushima Strait. In HFAc2-rcp85 and HFA-c0-rcp85, the typhoons changed their track towards the northeast in the vicinity of Shanghai which is located north of Taiwan. The experiment of HFA-c1-rcp85 had difficulty in identifying future typhoon features due to its limited typhoon events (i.e., only 7 typhoons). The future changes of the typhoon genesis locations between the present climate run and future climate runs with different SST conditions are predicted to range from -0.80 to $0.29^{\circ}$ and from 0.38 to $0.79^{\circ}$ for longitude and latitude, respectively (Supplementary Table 2). Except for HFA-c2-rcp85, the typhoon genesis locations are expected to shift east (north) between $0.38^{\circ}\left(0.31^{\circ}\right)$ and $0.79^{\circ}\left(0.80^{\circ}\right)$ for longitude (latitude). As for the typhoon lysis locations, the variation ranges from $1.90^{\circ}\left(0.93^{\circ}\right)$ to $2.44^{\circ}\left(1.40^{\circ}\right)$ for longitude (latitude). The degree of the changes in longitude is about 2 times greater than that in latitude. The typhoon lysis locations are predicted to move to the west (north) for longitude (latitude) in all experiments. The changes in the mean lysis locations are $2.19^{\circ}$ in longitude and $1.07^{\circ}$ in latitude.

Figure $2 \mathrm{f}, \mathrm{g}$, h represent comparisons of typhoon tracks from ensemble experiments under the different global warming scenarios with the same SST condition, c0. The typhoons routes of HFA with the different global warming scenarios tend to be similar to those of HPA,
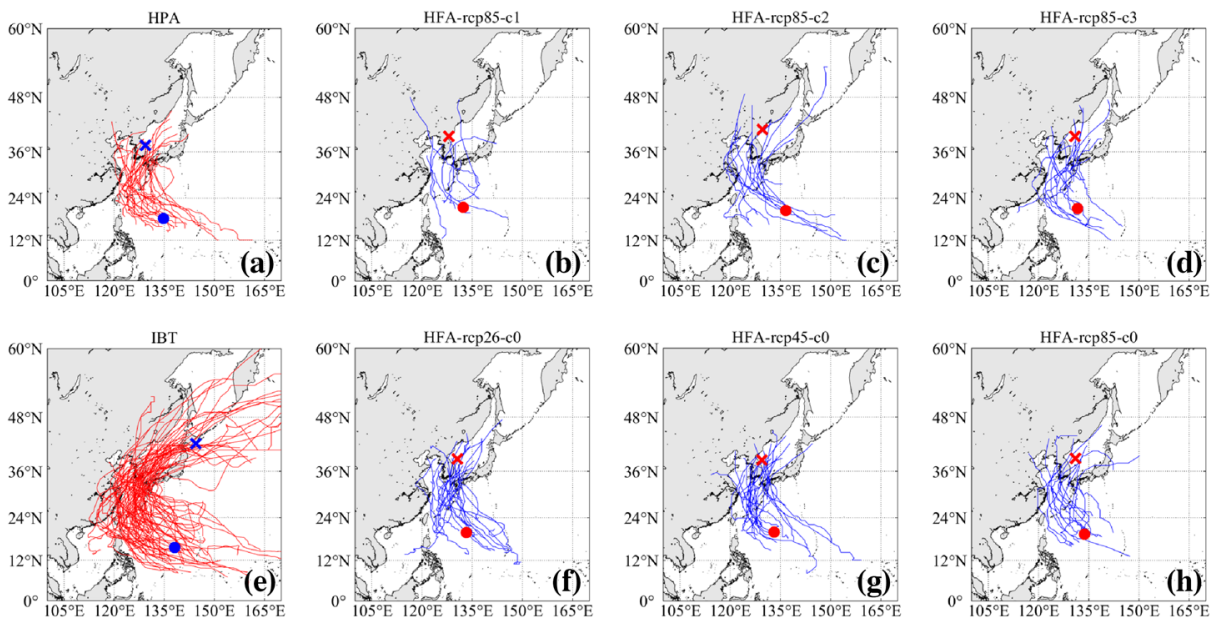

Fig. 2 Typhoon tracks of a simulation under the present climate condition (abbreviated as "HPA"), b projection under the RCP8.5 scenario using Cluster 1 of CMIP5 SST (abbreviated as “HFA- rcp85-c1"), c HFA-rcp85-c2, d HFA-rcp85-c3, e observations (IBTrACS) under the present climate condition, f projection under the rcp2.6 scenario using ensemble mean of CMIP5 SST experiments (abbreviated as "HFA-rcp26-c0"), g HFA-rcp45-c0 and (h) HFA-rcp85-c0. The circle and the cross on each panel indicate the average location of typhoon genesis and lysis, respectively 
whereas the future changes of the typhoon genesis locations between HPA and HFA have a different range of $-0.31^{\circ}\left(0.38^{\circ}\right)$ to $0.04^{\circ}\left(0.46^{\circ}\right)$ for longitude (latitude) depending on the global warming scenarios of HFA. In addition, typhoon lysis locations vary from $2.13^{\circ}\left(0.93^{\circ}\right)$ to $2.48^{\circ}\left(1.00^{\circ}\right)$ for longitude (latitude). Here, as stated above, the degree of the changes is about 2 times greater in longitude than in latitude. The typhoon lysis locations are predicted to shift to the east (north) for longitude (latitude) in all experiments. Especially, the tendency of future change of the lysis location with respect to latitude becomes more pronounced as the greenhouse gas concentration decreases. From the above results, it is anticipated that while the typhoon routes will not change significantly depending on the future climate conditions, genesis and lysis locations of the typhoons will move to the northwest and the northeast, respectively. The northward shift of the typhoon track, also referred to as the increase in typhoon track density along the north, over the KP shown here is consistent with the previous studies (e.g., Emanuel 2013; Manganello et al. 2014; Park et al. 2017; Nakamura et al. 2017).

\subsubsection{Typhoon intensity}

Finally, features of the typhoon intensity, which was expressed as a minimum central pressure of typhoons (MCP), were estimated. This study generated the wind field using a gradient wind field model for surge simulation with each typhoon event, so the characteristics of wind velocity of the typhoons are not considered here. Mean values of the probability density of MCP were $972.8 \mathrm{hPa}$ and $981.6 \mathrm{hPa}$ for HPA and IBTrACS, respectively (Supplementary Fig. 2). The mean value for HPA was smaller than that for IBTrACS by $8.8 \mathrm{hPa}$. Mori and Takemi (2016) indicate that a weak representation of the wall cloud makes MRI-AGCM3.2H yield a lower central air pressure. This difference of the mean values of MCP between HPA and IBTrACS, however, cannot be ignored in storm surge simulations (Harris 1963). As the points in the Q-Q plot of the mean values of MCP of HPA and IBTrACS approximately lie on a line, the MCP of HPA was linearly calibrated for all ensemble experiments: $\mathrm{MCP}_{\mathrm{HFA}}=\mathrm{MCP}_{\mathrm{HPA}}+$ $8.8 \mathrm{hPa}$. As a result of the calibration, the probability distribution of MCP for HPA corresponded well to one of IBTrACS.

The probability density function of MCP in the future climate varied with SST condition and global warming scenario condition (Supplementary Fig. 3). The frequency of highintensity typhoons with a central pressure lower than $930 \mathrm{hPa}$, which have not occurred so far, was forecasted to increase. Mean values of MCP in each experiment using the different global warming scenarios with the same SST condition, c0, were $981.6 \mathrm{hPa}, 974.0 \mathrm{hPa}$, $973.8 \mathrm{hPa}$, and $967.4 \mathrm{hPa}$, in HPA, HFA-rcp26, HFA-rcp45, and HFA-rcp85, respectively. The change rate for those in the future climate declined in the range of 0.8 to $1.4 \%$. The level of the change rate grows as the greenhouse gas concentration increases. The mean values of MCP in each experiment for the different SST condition under the same global warming scenario, RCP8.5 were $967.4 \mathrm{hPa}, 973.8 \mathrm{hPa}, 972.9 \mathrm{hPa}$, and $966.8 \mathrm{hPa}$ in HFA-c0, in HFA$\mathrm{c} 1$, in HFA-c2, in HFA-c3, respectively, and those change rate decreased from 1.3 to $1.4 \%$ in the future climate. These results suggest that the change rate of the mean values of MCP is greatly affected by global warming condition than SST condition. A summary of the typhoon characteristics of MCP for each ensemble experiment is given in Table 2.

In this section, the future changes of typhoon characteristics were examined using the typhoon data which is extracted from the climate experiment results. In the next section, future maximum SSHs around the KP coast are projected and its uncertainties are assessed using these typhoon data. 


\subsection{Changes in storm surge heights and those uncertainties}

Storm surge simulations targeting along the coast of the KP (D2) and the southeastern area of the KP (D4) were carried out to project future change of extreme SSHs using the typhoon with the bias correction for MCP. In addition, those uncertainties resulting from given global warming scenarios and spatial SST conditions were estimated. However, it is hard to evaluate an extreme $\mathrm{SSH}$ with a specific return period such as 50 years and 100 years using time-slice climate experiments with 20-50 years due to the lack of typhoon event (e.g., Nakajo et al. 2014; Kim et al. 2014). This study focused on maximum SSHs in D2 and higher percentile SSHs in D4 for the uncertainty assessment.

\subsection{Target area 1: the Korean Peninsula (D2)}

First, future change of maximum SSHs around the KP and its uncertainty were evaluated. Figure 3 present the spatial distributions of projected future change of maximum SSHs (Spatial distributions of maximum SSH and its prone area information under present and future climate conditions are provided in the Supplementary Fig. 4 and Supplementary Note 1, respectively.). The future change of maximum SSHs ranged from -2.03 to $5.52 \mathrm{~m}$ depending on the

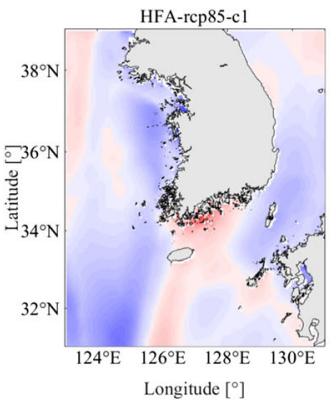

(a)

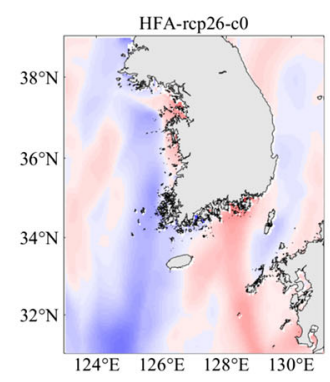

(d)

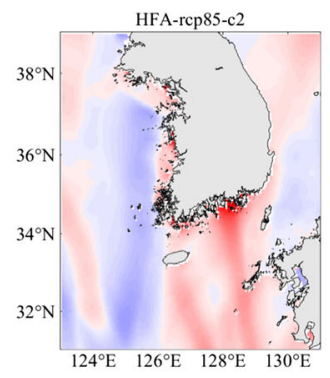

(b)

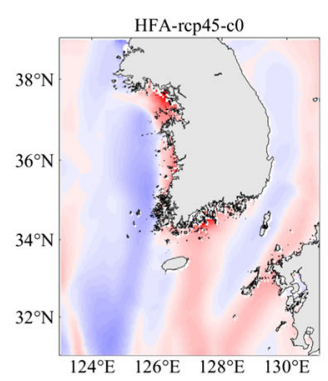

(e)

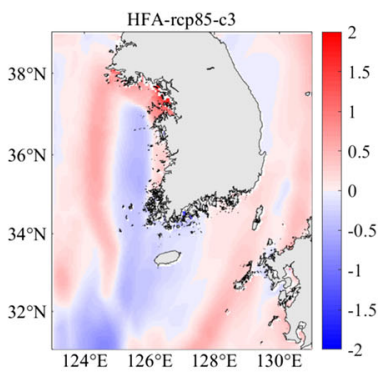

(c)

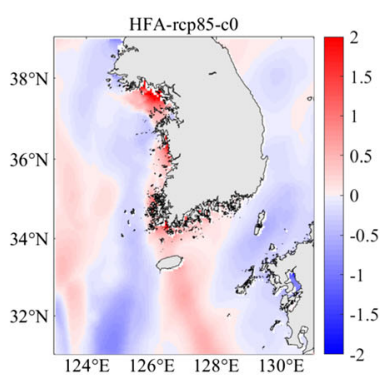

(f)

Fig. 3 Future change of projected maximum storm surge heights (unit: $m$ ) targeting the Korean Peninsula between an experiment under the present climate condition (abbreviated as "HPA") and an experiment a under the RCP8.5 scenario with cluster 1 of CMIP5 SST (abbreviated as "HFA-c1-rcp85"), b HFA-c2-rcp85, c HFAc3-rcp85, $\mathbf{d}$ under the rcp2.6 scenario with ensemble mean of CMIP5 SST experiments (abbreviated as "HFA-c0rcp26"), e HFA-c0-rcp45 and (f) HFA-c0-rcp85. Warm/cold colors indicate increase/decrease in the future climate, respectively. The letters in each panel indicate the city name. Refer to Fig. 1 for the meaning of each letter 
experiment condition. Table 3 shows vulnerable areas to storm surge, values of maximum $\mathrm{SSH}$, and ranges of its future change around the KP for each ensemble experiment.

The future changes of the maximum SSH for ensemble experiments using different SST patterns are presented in Fig. 3 a, b, c, and f. It is shown that those changes are relatively large in the regions where the high SSHs more than $2.0 \mathrm{~m}$ occurred in each climate experiment and are strongly influenced by some specific high-intensity typhoons. The distributions of future change of maximum SSHs showed similar patterns depending on the greenhouse gas concentration scenarios applied, but the range of the variation is different (Fig. $3 \mathrm{~d}$, e, and f). This result implies that future change of SSH can be predicted by applying pattern scaling between SSH and radiative forcing (e.g., Mitchell 2003; Mori et al. 2019). These results indicate that SST patterns and global warming scenarios would affect the typhoon characteristics in future conditions, which could accordingly change future maximum SSH and vulnerable area to storm surge. Because the number of typhoon events that can be considered in this study is limited, it is difficult to identify the contribution of each typhoon characteristic component to values of maximum SSH (refer to Supplementary Note 2 for detailed explanation). Here, we analyzed the contribution of the external meteorological force components of sea level pressure (SLP) and wind formed by the typhoon to maximum SSH.

The maximum SSH-induced by SLP of each climate experiment (Supplementary Fig. 5) was calculated by generating pressure fields at the time of the lowest central pressure for each typhoon event applying the Fujita model (1952) and by multiplying the pressure decrease (= $1013 \mathrm{hPa}$ - pressure) by $1 \mathrm{~cm}$. The maximum SSH-induced by wind was computed by subtracting the maximum SSH-induced by SLP from the total maximum SSH (Supplementary Fig. 4). Values less than zero were treated as zero. The maximum SSH-induced by SLP ranged from $0.55 \sim 0.86 \mathrm{~m}$ depending on the experiment conditions, whereas the maximum SSHinduced by wind is 0.75 to $6.61 \mathrm{~m}$ (Supplementary Table 3 ). For each experimental condition, SLP contributed about $0.2 \mathrm{~m}$ to the maximum SSH and no regional dependence was shown. By contrast, the maximum SSH induced by wind had a large regional dependence for each experimental condition, and the spatial distributions of total maximum SSH are similar to that

Table 3 Vulnerable coast areas to storm surge, maximum storm surge height and range of its future change depending on calculation domain for each ensemble experiment

Calculation domain

Climate

condition
Around the Korean Peninsula (KP, domain 2)
Around southeastern coast of the KP (domain 4)

$a^{*} \quad b^{*} \quad c^{*}$

\begin{tabular}{|c|c|c|c|c|c|c|c|}
\hline Present & HPA & W & 2.73 & - & W & 2.83 & - \\
\hline \multirow{6}{*}{ Future } & HFA-c0-rcp26 & $\mathrm{W}$ and SE & 3.62 & $-1.62 \sim 1.39$ & SE & 2.70 & $-2.26 \sim 2.23$ \\
\hline & HFA-c0-rcp45 & $\mathrm{W}$ and $\mathrm{S}$ & 4.64 & $-0.89 \sim 3.39$ & $\mathrm{~S}$ & 5.12 & $-0.30 \sim 3.72$ \\
\hline & HFA- c0-rcp85 & $\mathrm{W}$ and $\mathrm{S}$ & 4.87 & $-2.03 \sim 3.45$ & $\mathrm{~S}$ & 3.16 & $-0.52 \sim 2.12$ \\
\hline & HFA-c1-rcp85 & $\mathrm{S}$ & 2.64 & $-1.77 \sim 2.04$ & $\mathrm{~S}$ & 5.20 & $-0.83 \sim 2.49$ \\
\hline & HFA-c2-rcp85 & Whole & 4.41 & $-1.03 \sim 3.66$ & $\mathrm{~S}$ and $\mathrm{SE}$ & 5.49 & $-0.24 \sim 4.90$ \\
\hline & HFA-c3-rcp85 & $\mathrm{W}$ and SE & 3.26 & $-1.51 \sim 2.08$ & - & 1.14 & $-2.18 \sim 0.55$ \\
\hline
\end{tabular}

$\mathrm{a}^{*}$ : Vulnerable coast area with an increase in future change of storm surge height ( $W$ west coast, $S$ south coast, $S W$ southwest coast, $S E$ southeast coast)

$\mathrm{b}^{*}$ : Maximum storm surge height $(\mathrm{m})$

$\mathrm{c}^{*}$ : Range of future change of maximum storm surge height $(\mathrm{m})$. Negative and positive values mean decrease and increase, respectively 
of the maximum SSH induced by wind. For coastal areas in the KP, the ratio of maximum SSH induced by wind to the total maximum SSH was significantly higher than that by SLP (Supplementary Fig. 6). This study quantitatively evaluated the contribution of SLP and wind to the maximum SSH based on a limited number of typhoon event data. However, we did not use the SLP information at the occurrence time of the maximum SSH but used one at the time of the lowest SLP. That could create uncertainty in the contribution of each external force to the maximum SSH. Future research may reduce theses uncertainty through using a great deal of atmospheric information at the maximum SSH occurrence time and detailed statistical analysis.

Kim et al. (2014) assessed the future SSH around the KP using the typhoon data projected by the single climate experiment with the ensemble mean SST under the Special Report on Emissions Scenarios (SRES) A1B of the Coupled Model Intercomparison Project phase 3 (CMIP3) using MRI-AGCM with 20-km resolution. They suggested that the future change of the maximum SSH on the southwest coast is more than $1.0 \mathrm{~m}$, while it is less than $0.5 \mathrm{~m}$ on the west and southeast coasts and is relatively small on the east coast. It implies that the southwest coast is more vulnerable to future storm surge, which is in accordance with our findings for HFA-rcp-45-c0 and HFA-rcp85-c0. Therefore, our results can be considered reasonable because the radiative forcing of the SRES A1B scenario has a value similar to that of the RCP 6.0 scenario (van Vuuren and Carter 2013). However, a further review on the small future change of maximum SSHs on the west coast for their study would be necessary. In addition, we suppose that the discrepancies of future change of maximum SSHs between two studies result from the difference in the model grid resolution, greenhouse gas concentration scenario, bathymetric data, and so on. The significance here is that it was found that climate change scenarios are likely to affect the future change of maximum SSHs and the identification of vulnerable areas to storm surge.

\subsection{Target area 2: the southeastern region of the Korean Peninsula (D4)}

Future change of maximum SSHs in the KP's southeastern region in the higher resolution of D4 was assessed. Spatial distributions of projected future change of maximum SSHs for each ensemble experiment are shown in Fig. 4. (Spatial distributions of maximum SSH and its prone area information under present and future climate conditions are provided in the Supplementary Fig. 7 and Supplementary Note 3, respectively.) The extent of the future change of maximum SSH was from $-2.26 \mathrm{~m}$ to $4.90 \mathrm{~m}$.

Figure $4 \mathrm{a}, \mathrm{b}$, c, and $\mathrm{f}$ present the spatial distributions for the ensemble experiments using the different SST patterns. It indicates that the vulnerable areas and the magnitude of SSHs to the future condition were dependent on the chosen SST patterns. In HFA-rcp85-c1 and HFArcp85-c0, western parts including Boseong $(\mathrm{M})$, Goheung $(\mathrm{N})$, and Suncheon $(\mathrm{O})$ of southeastern coast was identified to be storm surge-prone areas. In contrast, future change of SSHs in the western part was expected to decrease under the HFA-c3-rcp85 condition. In HFArcp85-c2, the western part including Boseong $(\mathrm{M})$ and Goheung $(\mathrm{N})$ and the central area such as Sacheon (S), Namhae (R), and Tongyeong (U) were also predicted to be relatively prone. The most dangerous SST pattern on the southeastern coast was HFA-rcp85-c2 among the experiments. The future change of the maximum SSH was expected to increase across the whole southern coast of the KP in that case.

The spatial distributions for the ensemble experiments with the different global warming scenarios are shown in Fig. 4 d, e, and f. It can be seen that potential storm surge risks move 


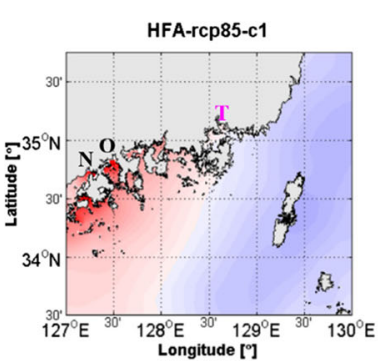

(a)

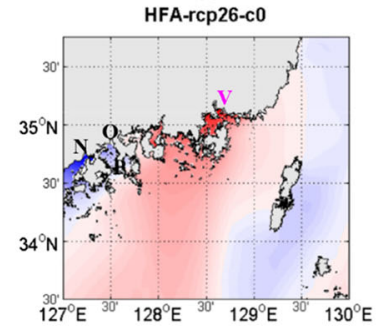

(d)

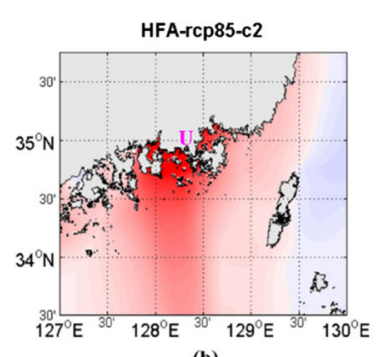

(b)

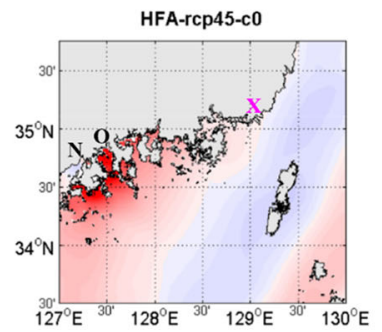

(e)

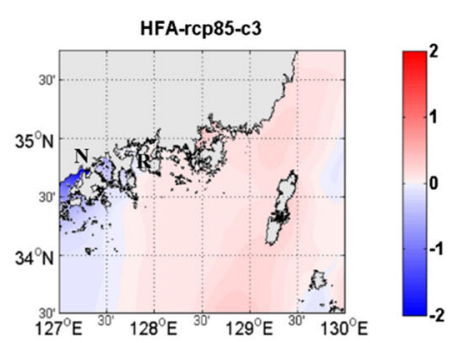

(c)

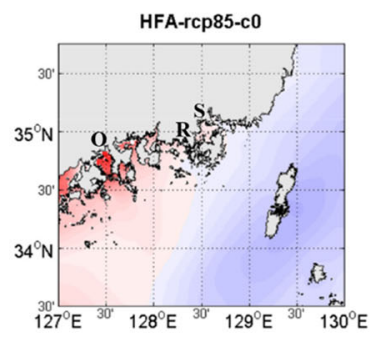

(f)

Fig. 4 Future change of maximum storm surge heights (unit: $\mathrm{m}$ ) around the southeastern region between an experiment under the present climate condition (abbreviated as "HPA") and an experiment a under the RCP8.5 scenario with cluster 1 of CMIP5 SST (abbreviated as "HFA-rcp8-c1"), b HFA-c2-rcp85, c HFA-c3-rcp85, d under the rcp2.6 scenario with ensemble mean of CMIP5 SST experiments (abbreviated as "HFA-c0-rcp26"), e HFA-c0-rcp45 and (f) HFA-c0-rcp85. Warm/cold colors indicate an increase/decrease in the future climate, respectively. The letters in each panel indicate the city name. Refer to Fig. 1 for the meaning of each letter

towards the western part of the southeastern coast. This tendency was in line with the trend of the future typhoon pathway presented in Fig. 2. Comparing future changes in SSHs at Goheung (N) based on future climate conditions (Fig. 5), its uncertainty tends to increase as greenhouse gas concentration increases. It is also different according to the SST condition, which produced the greatest uncertainty under the HFA-rcp85-c0 condition, while the lack of typhoon event in the HFA-rcp85-c1 experiment (i.e., only 7 typhoons) prevents its in-depth evaluation.

In this study, the storm surge heights were simulated using all typhoons extracted from the climate experiments under present and future climate conditions as external force. Therefore, it can be postulated that extracted typhoons and resulting storm surge heights are population data under specific climate conditions, not sampled data. This is why further statistical analysis was not implemented as we provided and analyzed entire sets of numerical results directly throughout the study. However, it is noted that the study is limited to the range of the future change of maximum SSH above, which would not be robust values. Robust value means that the value is not significantly affected by outliers or errors. The maximum SSH value appears to be greatly influenced by the characteristics of a few intensity typhoons. Still, it is tough to determine whether these values are outliers or not using current experimental data alone due to the deficiency of strengthening typhoon data. Thus, large-scale ensemble climate experiments would be necessary to overcome this shortcoming by securing a number of typhoon events with various typhoon characteristics (e.g., Mizuta et al. 2017) and statistical synthesis (e.g., Emanuel et al. 2006), and by projecting future change of extreme SSH using these abundant typhoon data (e.g., Yang et al. 2018; Bloemendaal et al. 2020). 


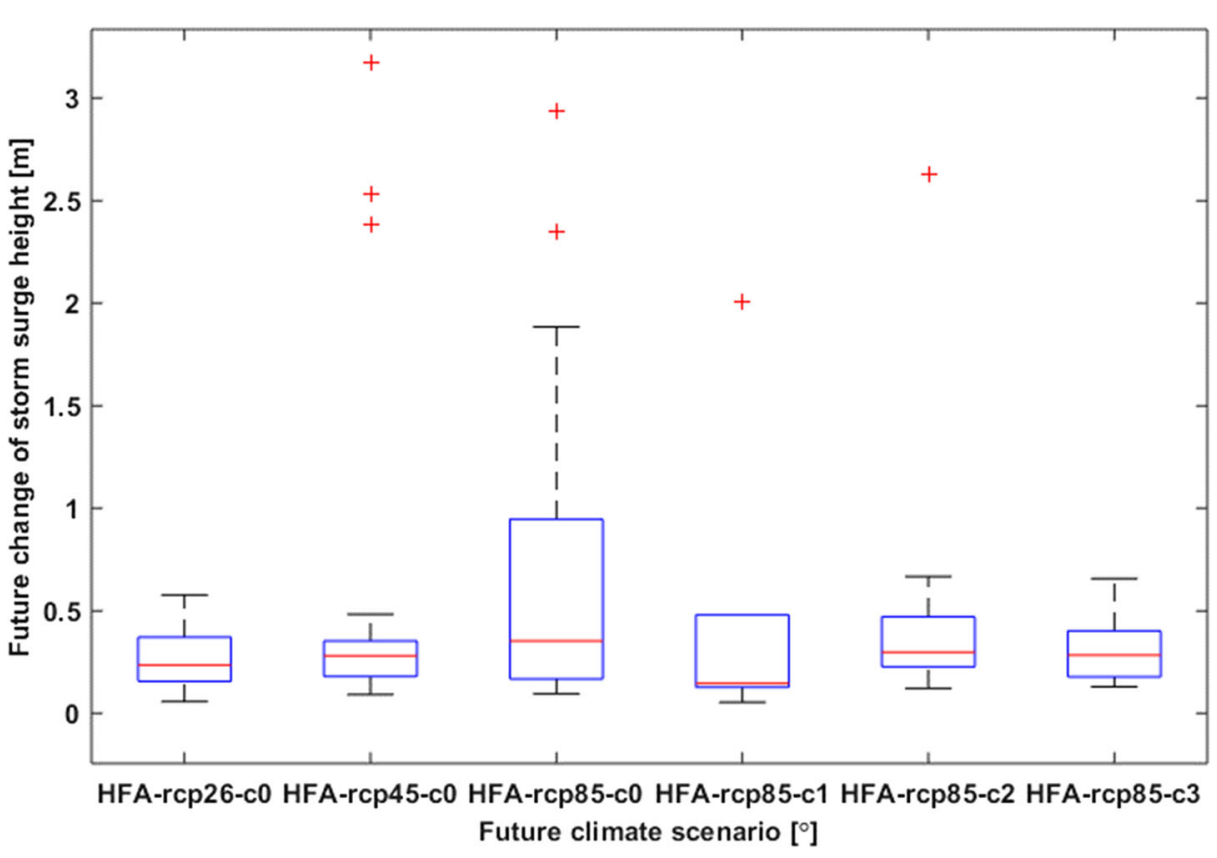

Fig. 5 Boxplot of future changes of storm surge height at Goheung (unit: $m$ ). The lower black, red and upper black lines indicate $25 \%, 50 \%$, and $75 \%$ quantile values, respectively. The cross marks mean outlier values

\section{Conclusions}

The present study assesses the uncertainties in projecting future change of extreme SSH. Storm surge simulations are conducted by using predicted typhoon data as an external force. The typhoon data is extracted from ensemble experiment results with three global warming scenarios and four sea surface temperature (SST) conditions using a single atmospheric general circulation model with $60-\mathrm{km}$ resolution. Here, we focused on typhoons passing in the vicinity of the Korean Peninsula (KP) which was defined as the region 32 to $40^{\circ} \mathrm{N}$ and 122 to $132^{\circ} \mathrm{E}$.

First, we examined the uncertainties of the projection of the typhoon properties such as the number, tracks and intensities based on projected typhoons by ensemble experiments. The reproducibility of typhoon was examined through a comparison between modeled typhoon data and observed one for the present climate condition. The number of typhoons for the present climate condition was found to be underestimated when compared with observed one, while the modeled typhoon tracks were well reproduced. Also, the intensity of modeled typhoons, which was expressed as a minimum central pressure (MCP) of the typhoons, was lower than observed one by $8.8 \mathrm{hPa}$. Because this bias cannot be ignored in simulating SSHs, the value of MCP from all ensemble experiments were linearly calibrated and then were used to simulate SSHs under present and future climate conditions.

Projection on future changes of typhoon properties and its uncertainties are discussed next. It is expected that the number of typhoons declined with a range from 4 to $73 \%$ and those intensities gathered strength in the range of 0.8 to $1.3 \%$. In addition, it is anticipated that the locations of genesis and lysis of the typhoon moved to the northwest and northeast for all ensemble experiments, respectively. However, the scale of each change is expected to alter according to global warming scenario and the conditions of SST pattern. 
Finally, simulations of storm surge targeting the KP were conducted using the typhoon data. The vulnerable area to future storm surge and the extent of future change of extreme SSHs depended on the spatial pattern of SST and the global warming scenario. Those will affect the typhoon characteristics in the future climate condition, which accordingly change future SSH. Especially, the distributions of future change of maximum SSHs depending on the greenhouse gas concentration scenarios show similar patterns, whereas the level of change is different. It implies that the future change of SSH can be predicted by applying pattern scaling between SSH and radiative forcing. With regard to uncertainty in projecting future change of $\mathrm{SSH}$, the uncertainty tends to increase as greenhouse gas concentration increases, but does not show similarity with respect to SST condition.

These conclusions above, however, are highly uncertain due to insufficient TC event from the climate ensemble experiments. It is thought to be meaningful that the SST and global warming scenarios have been qualitatively evaluated for the impact on the future typhoon characteristics. It would be necessary to project future extreme SSH based on a large amount of $\mathrm{TC}$ events in order to reduce the uncertainties.

Acknowledgments We thank the anonymous reviewers for their helpful comments.

Author attribution All authors contributed to the study conception and design. Material preparation, data collection, and analysis were performed by Nobuhito Mori and Jung-A Yang. The first draft of the manuscript was written by Jung-A Yang and all authors commented on previous versions of the manuscript. All authors read and approved the final manuscript.

Funding information This research was supported by Basic Science Research Program through the National Research Foundation of Korea (NRF) funded by the Ministry of Education(2019R1I1A1A01064178), by the Integrated Research Program for Advancing Climate Models (TOUGOU Program, JPMXD0717935498) and by Grant-in-Aid for Scientific Research (KAKENHI) supported by the Ministry of Education, Culture, Sports, Science and Technology (MEXT), Japan.

\section{Compliance with ethical standards}

Conflict of interest The authors declare that they have no conflict of interest.

Open Access This article is licensed under a Creative Commons Attribution 4.0 International License, which permits use, sharing, adaptation, distribution and reproduction in any medium or format, as long as you give appropriate credit to the original author(s) and the source, provide a link to the Creative Commons licence, and indicate if changes were made. The images or other third party material in this article are included in the article's Creative Commons licence, unless indicated otherwise in a credit line to the material. If material is not included in the article's Creative Commons licence and your intended use is not permitted by statutory regulation or exceeds the permitted use, you will need to obtain permission directly from the copyright holder. To view a copy of this licence, visit http://creativecommons.org/licenses/by/4.0/.

\section{References}

Bengtsson L, Hodges KI, Esch M et al (2007) How may tropical cyclones change in a warmer climate? Tellus A 59:539-561. https://doi.org/10.1111/j.1600-0870.2007.00251.xzZzZZ

Bloemendaal N, Haigh ID, de Moel H et al (2020) Generation of a global synthetic tropical cyclone hazard dataset using STORM. Sci Data. 7:40. https://oi.org/10.1038/s41597-020-0381-27

Buser CM, Künsch HR, Lüthi D et al (2009) Bayesian multi-model projection of climate: bias assumptions and interannual variability. Climate Dyn 33:849-868. https://doi.org/10.1007/s00382-009-0588-6 
Ceres RL, Forest CE, Keller K (2017) Understanding the detectability of potential changes to the 100-year peak storm surge. Clim Chang 145:221-235

Christensen JH, Kanikicharla KK, Aldrian E et al (2013) Climate phenomena and their relevance for future regional climate change. In: Stocker TF et al (eds) Climate change 2013: the physical science basis. Contribution of working group I to the fifth assessment report of the intergovernmental panel on climate change. Cambridge University Press, Cambridge, pp 1217-1308. https://doi.org/10.1017 /CBO9781107415324.028

Emanuel KA (2013) Downscaling CMPI5 climate models shows increased tropical cyclone activity over the 21st century. Proc Natl Acad Sci U S A 110:12219-12224. https://doi.org/10.1073/pnas.1301293110

Emanuel K, Ravela S, Vivant E et al (2006) Statistical deterministic approach to hurricane risk assessment. Bull. Am. Meteor. Soc. 87:299-314. https://doi.org/10.1175/BAMS-87-3-299

Endo H, Kitoh A, Ose T et al (2012) Future changes and uncertainties in Asian precipitation simulated by multiphysics and multi-sea surface temperature ensemble experiments with high-resolution Meteorological Research Institute atmospheric general circulation models (MRI-AGCMs). J Geophys Res 117:D16118. https://doi.org/10.1029/2012JD017874

Fujii T, Mitsuta Y (1986) Synthesis of a stochastic typhoon model and simulation of typhoon winds. Annu Disas Prev Res Inst Kyoto Univ:229-239

Fujita T (1952) Pressure distribution within typhoon. Geophys Mag 23:437-451

Harris DL (1963) Characteristics of the hurricane strom surge. Department of Commerce, Weather Bureau, Washington D C, p 139

Hur D, Yeom G, Kim J et al (2006) Estimation of storm surges on the coast of Busan. J Ocean Eng Technol 20: 37-44

IPCC, 2019: Summary for policymakers. In: IPCC special report on the ocean and cryosphere in a changing climate [H.-O. Pörtner, D.C. Roberts, V. Masson-Delmotte, P. Zhai, M. Tignor, E. Poloczanska, K. Mintenbeck, A. Alegría, M. Nicolai, A. Okem, J. Petzold, B. Rama, N.M. Weyer (eds.)]. In press

Kim SY, Yasuda T, Mase H (2008) Numerical analysis of effects of tidal variations on storm surges and waves. Appl Ocean Res 30:311-322. https://doi.org/10.1016/j.apor.2009.02.003

Kim SY, Oh J, Suh KD et al (2014) Evaluation of storm surges around the Korean Peninsula in present and future climates. In: Coastal Engineering Proceedings. p 23

Kitoh A, Endo H (2016) Changes in precipitation extremes projected by a $20-\mathrm{km}$ mesh global atmospheric model. Weather Clim Extrem 11:41-52. https://doi.org/10.1016/j.wace.2015.09.001

Kitoh A, Ose T, Takayabu I (2016) Dynamical downscaling for climate projection with high-resolution MRI AGCM-RCM. J Meteorol Soc Japan Ser II 94:1-16. https://doi.org/10.2151/jmsj.2015-022

Knapp KR, Kruk MC, Levinson DH et al (2010) The international best track archive for climate stewardship (IBTrACS) unifying tropical cyclone data. Bull Am Meteorol Soc 91:363-376. https://doi.org/10.1175/2009 BAMS2755.1

Lapidez JP, Tablazon J, Dasallas L et al (2015) Identification of storm surge vulnerable areas in the Philippines through the simulation of Typhoon Haiyan-induced storm surge levels over historical storm tracks. Nat Hazards Earth Syst Sci 15:1473-1481. https://doi.org/10.5194/nhess-15-1473-2015

Lin N, Marsooli R, Colle BA (2019) Storm surge return levels induced by mid-to-late-twenty-first-century extratropical cyclones in the Northeastern United States. Clim Chang 154:143-158

Manganello JV, Hodges KI, Dirmeyer B et al (2014) Future change in the Western North Pacific tropical cyclone activity projected by a multidecadal simulation with a 16-km global atmospheric GCM. J Clim 27:76227646. https://doi.org/10.1175/JCLI-D-13-00678.1

Ministry of Oceans and Fisheries (2017) Design manual for port and harbour facilities in Korea (in Korean)

Mitchell TD (2003) Pattern scaling: an examination of the accuracy of the technique for describing future climates. Clim Chang 60:217-242. https://doi.org/10.1023/A:1026035305597

Mizuta R, Yoshimura H, Murakami H et al (2012) Climate simulations using MRI-AGCM3. 2 with 20-km grid. J Meteorol Soc Japan Ser II 90:233-258. https://doi.org/10.2151/jmsj.2012-A12

Mizuta R, Arakawa O, Ose T et al (2014) Classification of CMIP5 future climate responses by the tropical sea surface temperature changes. SOLA 10:167-171. https://doi.org/10.2151/sola.2014-035

Mizuta R, Murata A, Ishi M et al (2017) Over 5,000 years of ensemble future climate simulations by $60-\mathrm{km}$ global and 20-km regional atmospheric models. Bull. Am. Meteor. Soc. 98:1383-1398. https://doi. org/10.1175/BAMS-D-16-0099.1

Mori N, Takemi T (2016) Impact assessment of coastal hazards due to future changes of tropical cyclones in the North Pacific Ocean. Weather Clim Extrem 11:53-69. https://doi.org/10.1016/j.wace.2015.09.002

Mori N, Shimura T, Yoshida K et al (2019) Future changes in extreme storm surges based on mega-ensemble projection using 60-km resolution atmospheric global circulation model. Coast Eng J 61:295-307

Murakami H, Sugi M (2010) Effect of model resolution on tropical cyclone climate projections. Sola 6:73-76. https://doi.org/10.2151/sola.2010-019 
Murakami H, Mizuta R, Shindo E (2012a) Future changes in tropical cyclone activity projected by multi-physics and multi-SST ensemble experiments using the 60-km-mesh MRI-AGCM. Clim Dyn 39:2569-2584. https://doi.org/10.1007/s00382-011-1223-x

Murakami H, Wang Y, Yoshimura H et al (2012b) Future changes in tropical cyclone activity projected by the new high-resolution MRI-AGCM. J Clim 25:3237-3260. https://doi.org/10.1175/JCLI-D-11-00415.1

Nakajo J, Mori N, Yasuda T et al (2014) Global stochastic tropical cyclone model based on principal component analysis and cluster analysis. J Appl Meteorol Climatol 53:1547-1577. https://oi.org/10.1175/JAMC-D-13-08.1

Nakamura J, Camargo SJ, Sobel AH et al (2017) Western North Pacific tropical cyclone model tracks in present and future climates. J Geophys Res Atmos 122:9721-9744. https://doi.org/10.1002/2017JD027007

Park J, Obeysekera J, Irizarry M et al (2011) Storm surge projections and implications for water management in South Florida. Clim Chang 107:109-128

Park DSR, Ho CH, Chan JCL et al (2017) Asymmetric response of tropical cyclone activity to global warming over the North Atlantic and western North Pacific from CMPI5 model projections. Sci Rep 7:41354. https://doi.org/10.1038/srep41354

Pickering MD, Horsburgh KJ, Blundell JR et al (2017) The impact of future sea-level rise on the global tides. Cont Shelf Res 142:50-68. https://doi.org/10.1016/j.csr.2017.02.004

Ren D, Lynch M, Leslie LM et al (2014) Sensitivity of tropical cyclone tracks and intensity to ocean surface temperature: four cases in four different basins. Tellus A 66:24212. https://doi.org/10.3402/tellusa.v66.24212

Seo SN (2008) Digital 30sec gridded bathymetric data of Korea marginal seas-KorBathy30s. J Korean Soc Coast Ocean Eng 20:110-120

Stowasser M, Wang Y, Hamilton K (2007) Tropical cyclone changes in the western North Pacific in a global warming scenario. J Clim 20:2378-2396. https://oi.org/10.1175/JCLI4126.1

Troccoli A, Zambon F, Hodges KI et al (2012) Storm surge frequency reduction in Venice under climate change. Clim Chang 113:1065-1079

van Vuuren DP, Carter TR (2013) Climate and socio-economic scenarios for climate change research and assessment: reconciling the new with the old. Clim Chang 122:415-429. https://doi.org/10.1007/s10584013-0974-2

van Vuuren DP, Edmonds J, Kainuma M et al (2011) The representative concentration pathways: an overview. Clim Chang 109:5-31. https://doi.org/10.1007/s10584-011-0148-Z

Vousdoukas MI, Voukouvalas E, Annunziato A et al (2016) Projections of extreme storm surge levels along Europe. Clim Dyn 47:3171-3190. https://doi.org/10.1007/s00382-016-3019-5

Walsh K, Lavender S, Murakami H et al (2010) The tropical cyclone climate model intercomparison project. In: Elsner JB, Jagger TH (eds) Hurricanes and climate change. Springer, Berlin, pp 1-24

Yang SK, Kim SB (2014) A height simulation on storm surges in Jeju Island. J Environ Sci Int 23:459-472. https://doi.org/10.5322/JESI.2014.23.3.459

Yang JA, Kim S, Mori N, Mase H (2017) Bias correction of simulated storm surge height considering coastline complexity. Hydrological Research Letters 11:121-127. https://doi.org/10.3178/hrl.11.121

Yang JA, Kim S, Mori N, Mase H (2018) Assessment of long-term impact of storm surges around the Korean peninsula based on a large ensemble of climate projections. Coast Eng 142:1-8. https://doi.org/10.1016/j. coastaleng.2018.09.008

Yasuda T, Hayashi Y, Mori N et al (2010) A stochastic typhoon model applicable to storm surge and wave simulations for climate change projection. J Japan Soc Civ Eng Ser B2 (Coastal Eng) 66:1241-1245. https://doi.org/10.2208/kaigan.66.1241

Publisher's note Springer Nature remains neutral with regard to jurisdictional claims in published maps and institutional affiliations.

\section{Affiliations}

\section{Jung-A Yang ${ }^{1,4} \cdot$ Sooyoul $\mathrm{Kim}^{2} \cdot$ Sangyoung Son ${ }^{3} \cdot$ Nobuhito Mori $^{4} \cdot$ Hajime Mase $^{4}$}

1 Research Institute for Mega Construction, Korea University, 145 Anamro, Sungbuk-gu, Seoul 02841, Republic of Korea

2 Graduate School of Engineering, Tottori University, 4-101 Koyama-Minami, Tottori 680-8552, Japan

3 School of Civil, Environmental and Architectural Engineering, Korea University, 145 Anamro, Sungbukgu, Seoul 02841, Republic of Korea

4 Disaster Prevention Research Institute, Kyoto University, Gokasho, Uji, Kyoto 611-0011, Japan 